\title{
Optimal Bounds for Toader-Type Mean in Terms of Arithmetic and Centroidal Means
}

\author{
Xiao-Hong He${ }^{1}$, Yue-Ying Yang ${ }^{2 *}$, Ping $\mathrm{Ma}^{2}$, Wei-Mao Qian ${ }^{4}$ \\ ${ }^{I}$ Office of Academic Affairs, Quzhou Broadcast and TV University, Quzhou 324000, China. \\ ${ }^{2}$ School of Mechanical and Electrical Engineering, Huzhou Vocational \& Technical College, Huzhou 313000, \\ China. \\ ${ }^{3}$ Wei-Mao Qian, School of Distance Education, Huzhou Broadcast and TV University, Huzhou 313000, China. \\ *Corresponding Author: Yue-Ying Yang, School of Mechanical and Electrical Engineering, Huzhou \\ Vocational \& Technical College, Huzhou 313000, China.
}

Abstract: In this paper, we find the best possible parameters $\alpha_{1}, \alpha_{2}, \beta_{1}, \beta_{2} \in i_{\text {and }} \alpha_{3}, \beta_{3} \in[1 / 2,1]_{\text {Such that }}$ the double inequalities

$$
\begin{aligned}
& E^{\alpha_{1}}(a, b) A^{1-\alpha_{1}}(a, b)<T[A(a, b), Q(a, b)]<E^{\beta_{1}}(a, b) A^{1-\beta_{1}}(a, b), \\
& \alpha_{2} E(a, b)+\left(1-\alpha_{2}\right) A(a, b)<T[A(a, b), Q(a, b)]<\beta_{2} E(a, b)+\left(1-\beta_{2}\right) A(a, b), \\
& E\left[\alpha_{3} a+\left(1-\alpha_{3}\right) b, \alpha_{3} b+\left(1-\alpha_{3}\right) a\right]<T[A(a, b), Q(a, b)]<E\left[\beta_{3} a+\left(1-\beta_{3}\right) b, \beta_{3} b+\left(1-\beta_{3}\right) a\right] \\
& \text { hold for all } a, b>0_{\text {with }} a \neq b, \text { where } A(a, b)=(a+b) / 2, Q(a, b)=\sqrt{\left(a^{2}+b^{2}\right) / 2}, \\
& E(a, b)=2\left(a^{2}+a b+b^{2}\right) /[3(a+b)] \text { and } T(a, b)=\frac{2}{\pi} \int_{0}^{\pi / 2} \sqrt{a^{2} \cos ^{2} t+b^{2} \sin ^{2} t} d t \text { denote the }
\end{aligned}
$$

arithmetic, quadratic, centroidal and Toader means of two positive numbers $a_{\text {and }} b$, respectively.

Keywords: Toader mean, arithmetic mean, quadratic mean, centroidal mean, complete elliptic integral

2010 Mathematics Subject Classification: 26E60, $33 E 05$.

\section{INTRODUCTION}

For $p \in \mathbf{R}$ and $a, b>0$ with $a \neq b$, the $p$-th power mean $M_{p}(a, b)[1,2]$, harmonic mean $H(a, b)$, geometric mean $G(a, b)$, arithmetic mean $A(a, b)$, quadratic mean $Q(a, b)$, contra-harmonic mean $C(a, b)$, centroidal mean $E(a, b)$ and Toader mean $T(a, b)$ [3] are respectively defined by

$$
\begin{aligned}
& M_{p}(a, b)=\left[\left(a^{p}+b^{p}\right) / 2\right]^{1 / p}(p \neq 0), M_{0}(a, b)=\sqrt{a b}, \\
& H(a, b)=\frac{2 a b}{a+b}, G(a, b)=\sqrt{a b}, A(a, b)=\frac{a+b}{2}, \\
& Q(a, b)=\sqrt{\frac{a^{2}+b^{2}}{2}}, C(a, b)=\frac{a^{2}+b^{2}}{a+b},
\end{aligned}
$$


$E(a, b)=\frac{2\left(a^{2}+a b+b^{2}\right)}{3(a+b)}, T(a, b)=\frac{2}{\pi} \int_{0}^{\pi / 2} \sqrt{a^{2} \cos ^{2} t+b^{2} \sin ^{2} t} d t$.

It is well known that $M_{p}(a, b)$ is strictly increasing with respect to $p \in \mathbf{R}$ for fixed $a, b>0$ with $a \neq b$, symmetric and homogeneous of degree 1 , and the inequalities

$$
\begin{aligned}
& H(a, b)=M_{-1}(a, b)<G(a, b)=M_{0}(a, b)<A(a, b)=M_{1}(a, b) \\
& <T(a, b)<E(a, b)<Q(a, b)=M_{2}(a, b)<C(a, b)
\end{aligned}
$$

hold for all $a, b>0$ with $a \neq b$.

Let $r \in(0,1)$. Then the elliptic integral of the first kind $\kappa(r)$ and second kind $\varepsilon(r)$

$[4,5]$ are given by

$$
\begin{aligned}
& \kappa(r)=\int_{0}^{\pi / 2}\left(1-r^{2} \sin ^{2} t\right)^{-1 / 2} d t \\
& \varepsilon(r)=\int_{0}^{\pi / 2}\left(1-r^{2} \sin ^{2} t\right)^{1 / 2} d t
\end{aligned}
$$

respectively. We clearly see that the function $r$ a $\kappa(r)$ is strictly increasing from $(0,1)$ onto $(\pi / 2,+\infty)$ and the function $r$ a $\varepsilon(r)$ is strictly decreasing from $(0,1)$ onto $(1, \pi / 2)$, and they satisfy the formulas (See [6, Appendix E, pp. 474-475]).

$$
\begin{aligned}
& \frac{d \kappa(r)}{d r}=\frac{\varepsilon(r)-\left(1-r^{2}\right) \kappa(r)}{r\left(1-r^{2}\right)}, \frac{d \varepsilon(r)}{d r}=\frac{\varepsilon(r)-\kappa(r)}{r}, \\
& \frac{d\left[\varepsilon(r)-\left(1-r^{2}\right) \kappa(r)\right]}{d r}=r \kappa(r), \frac{d[\kappa(r)-\varepsilon(r)]}{d r}=\frac{r \varepsilon(r)}{1-r^{2}},
\end{aligned}
$$

the values $\kappa(\sqrt{2} / 2)$ and $\varepsilon(\sqrt{2} / 2)$ can be expressed as [7]

$$
\kappa\left(\frac{\sqrt{2}}{2}\right)=\frac{\Gamma^{2}(1 / 4)}{4 \sqrt{\pi}}=1.85407467 \cdots, \varepsilon\left(\frac{\sqrt{2}}{2}\right)=\frac{4 \Gamma^{2}(3 / 4)+\Gamma^{2}(1 / 4)}{8 \sqrt{\pi}}=1.35064388 \cdots
$$

where $\Gamma(x)=\int_{0}^{\infty} x^{x-1} e^{-t} d t$ is Euler gamma function.

The Toader mean $T(a, b)$ is well known in mathematical literature for many years, it satisfies

$$
T(a, b)=R_{E}\left(a^{2}, b^{2}\right)
$$

where

$$
R_{E}(a, b)=\frac{1}{\pi} \int_{0}^{+\infty} \frac{[a(t+b)+b(t+a)] t}{(t+a)^{3 / 2}(t+b)^{3 / 2}} d t
$$

stands for the symmetric complete elliptic integral of the second kind (see [8-10]), therefore it cannot be expressed in terms of the elementary transcendental functions, and the Toader mean $T(a, b)$ can be rewritten as 
$T(a, b)=\left\{\begin{array}{l}\frac{2 a}{\pi} \varepsilon\left(\sqrt{1-(b / a)^{2}}\right), a>b, \\ \frac{2 b}{\pi} \varepsilon\left(\sqrt{1-(a / b)^{2}}\right), a<b .\end{array}\right.$

Recently, the Toader mean has been the subject of intensive research. In particular, many remarkable inequalities for Toader mean can be found in the literature $[4,55,11-16]$.

Vuorinen [17] conjectured that

$M_{3 / 2}(a, b)<T(a, b)$

for all $a, b>0$ with $a \neq b$. This conjecture was proved by Qiu and Shen [18], and Barnard et al. [19], respectively.

Alzer and Qiu [20] presented the best possible upper power mean bound for the Toader mean as follows:

$T(a, b)<M_{\log 2 / \log (\pi / 2)}(a, b)$

for all $a, b>0$ with $a \neq b$.

Hua and Qi [21,22] proved that the double inequalities

$$
\begin{aligned}
& \alpha E(a, b)+(1-\alpha) A(a, b)<T(a, b)<\beta E(a, b)+(1-\beta) A(a, b) \\
& E[\lambda a+(1-\lambda) b, \lambda b+(1-\lambda) a]<T(a, b)<E[\mu a+(1-\mu) b, \mu b+(1-\mu) a]
\end{aligned}
$$

hold for all $a, b>0$ with $a \neq b$ if and only if $\alpha \leq 3 / 4, \beta \geq 12 / \pi-3, \lambda \leq(1+\sqrt{3} / 2) / 2$ and $\mu \geq 1 / 2+\sqrt{12 / \pi-3} / 2$.

$\mathrm{Xu}$ and Qian [23] present the best possible parameters $\alpha_{1} \leq 3 / 4, \beta_{1} \geq 2 \varepsilon(\sqrt{2} / 2) / \pi$

$=0.8598 \mathrm{~L}, \alpha_{2} \leq 5 / 6, \beta_{2} \geq 2 \varepsilon(\sqrt{2} / 2) / \pi=0.8598 \mathrm{~L}, \alpha_{3} \leq \sqrt{2} \varepsilon(\sqrt{2} / 2) / \pi=0.6080 \mathrm{~L}$ and $\beta_{3} \geq 5 / 8$ such that the double inequalities

$$
\begin{aligned}
& \alpha_{1} Q(a, b)+\left(1-\alpha_{1}\right) G(a, b)<T[A(a, b), Q(a, b)]<\beta_{1} Q(a, b)+\left(1-\beta_{1}\right) G(a, b), \\
& \alpha_{2} Q(a, b)+\left(1-\alpha_{2}\right) H(a, b)<T[A(a, b), Q(a, b)]<\beta_{2} Q(a, b)+\left(1-\beta_{2}\right) H(a, b), \\
& \alpha_{3} C(a, b)+\left(1-\alpha_{3}\right) H(a, b)<T[A(a, b), Q(a, b)]<\beta_{3} C(a, b)+\left(1-\beta_{3}\right) H(a, b)
\end{aligned}
$$

hold for all $a, b>0$ with $a \neq b$.

In [24], the authors prove that the double inequalities

$$
\begin{aligned}
& {\left[\alpha(r) A^{r}(a, b)+(1-\alpha(r)) Q^{r}(a, b)\right]^{1 / r}<T[A(a, b), Q(a, b)]} \\
& <\left[\beta(r) A^{r}(a, b)+(1-\beta(r)) Q^{r}(a, b)\right]^{1 / r}
\end{aligned}
$$

hold for all $r \leq 1$ and $a, b>0$ with $a \neq b$.

Chu et. al. [25] proved that the double inequalities

$$
Q\left[\lambda_{1} a+\left(1-\lambda_{1}\right) b, \lambda_{1} b+\left(1-\lambda_{1}\right) a\right]<T[A(a, b), Q(a, b)]<Q\left[\mu_{1} a+\left(1-\mu_{1}\right) b, \mu_{1} b+\left(1-\mu_{1}\right) a\right],
$$


$C\left[\lambda_{2} a+\left(1-\lambda_{2}\right) b, \lambda_{2} b+\left(1-\lambda_{2}\right) a\right]<T[A(a, b), Q(a, b)]<C\left[\mu_{2} a+\left(1-\mu_{2}\right) b, \mu_{2} b+\left(1-\mu_{2}\right) a\right]$

hold for all $r \leq 1$ and $a, b>0$ with $a \neq b$ if and only if $\lambda_{1} \leq 1 / 2+\sqrt{2 \varepsilon^{2}(\sqrt{2} / 2) / \pi^{2}-1 / 4}$

$=0.8459 \mathrm{~L}, \mu_{1} \geq 1 / 2+\sqrt{2} / 4, \lambda_{2} \leq 1 / 2+\sqrt{\sqrt{2} \varepsilon(\sqrt{2} / 2) /(2 \pi)-1 / 4}=0.7323 \mathrm{~L}$ and $\mu_{2} \geq 3 / 4$

For fixed $a, b>0$ with $a \neq b$, let $x \in[1 / 2,1], f(x)=E[x a+(1-x) b, x b+(1-x) a]$.

Then it is not difficult to verify that $f(x)$ is continuous and strictly increasing on $[0,1 / 2]$.

Note that

$f(1 / 2)=A(a, b)<T[A(a, b), Q(a, b)]<E(a, b)=f(1)$.

Motivated by inequalities (1.2) and (1.4), it is natural to ask what are the best possible parameters $\alpha_{1}, \alpha_{2}, \beta_{1}, \beta_{2} \in(0,1)$ and $\alpha_{3}, \beta_{3} \in[1 / 2,1]$ such that the double inequalities

$$
\begin{aligned}
& E^{\alpha_{1}}(a, b) A^{1-\alpha_{1}}(a, b)<T[A(a, b), Q(a, b)]<E^{\beta_{1}}(a, b) A^{1-\beta_{1}}(a, b), \\
& \alpha_{2} E(a, b)+\left(1-\alpha_{2}\right) A(a, b)<T[A(a, b), Q(a, b)]<\beta_{2} E(a, b)+\left(1-\beta_{2}\right) A(a, b), \\
& E\left[\alpha_{3} a+\left(1-\alpha_{3}\right) b, \alpha_{3} b+\left(1-\alpha_{3}\right) a\right]<T[A(a, b), Q(a, b)]<E\left[\beta_{3} a+\left(1-\beta_{3}\right) b, \beta_{3} b+\left(1-\beta_{3}\right) a\right]
\end{aligned}
$$

hold for all $a, b>0$ with $a \neq b$ ? The main purpose of this paper is to answer this question.

\section{LEMMAS}

In order to prove our main results we need some Lemmas, which we present in this section.

Lemma 2.1 (See [4], Theorem 1.25) Let $-\infty<a<b<+\infty, f, g:[a, b] \rightarrow \mathbf{R}$ be continuous on $[a, b]$ and differentiable on $(a, b)$, and $g^{\prime}(x) \neq 0$ on $(a, b)$. If $f^{\prime}(x) / g^{\prime}(x)$ is increasing (decreasing) on $(a, b)$, then so are the function

$[f(x)-f(a)] /[g(x)-g(a)]$ and $[f(x)-f(b)] /[g(x)-g(b)]$. If $f^{\prime}(x) / g^{\prime}(x)$ is strictly monotone, then the monotonicity in the conclusion is also strict.

Lemma 2.2 (1). The function $r$ a $\left[\varepsilon(r)-\left(1-r^{2}\right) \kappa(r)\right] / r^{2}$ is strictly increasing from $(0,1)$ onto $(\pi / 4,1)$.

(2) The function $r$ a $[\kappa(r)-\varepsilon(r)] / r^{2}$ is strictly increasing from $(0,1)$ onto $(\pi / 4,+\infty)$.

(3) The function $r$ a $\varepsilon(r) / \sqrt{3-2 r^{2}}$ is strictly increasing from $(0, \sqrt{2} / 2)$ onto $(\sqrt{3} \pi / 6, \varepsilon(\sqrt{2} / 2) / \sqrt{2})$.

(4) The function $r$ a $\sqrt{3-2 r^{2}}\left[\varepsilon(r)-\left(1-r^{2}\right) \kappa(r)\right] / r^{2}$ is strictly decreasing from $(0, \sqrt{2} / 2)$ onto $(\sqrt{2}(2 \varepsilon(\sqrt{2} / 2)-\kappa(\sqrt{2} / 2)), \sqrt{3} \pi / 4)$. 
Proof. Parts (1) and (2) can be found in [6 Theorem 3.21(1), Exercise 3.43(11)].

For part (3), Let $\phi(r)=\varepsilon(r) / \sqrt{3-2 r^{2}}$. Then simple computations lead to

$\phi\left(0^{+}\right)=\frac{\sqrt{3} \pi}{6}, \phi\left(\sqrt{2} / 2^{-}\right)=\sqrt{2} \varepsilon(\sqrt{2} / 2) / 2$,

Differentiating $\phi(r)$ gives

$$
\begin{aligned}
& \phi^{\prime}(r)=\frac{3 \varepsilon(r)-3 \kappa(r)+2 r^{2} \kappa(r)}{r\left(3-2 r^{2}\right)^{3 / 2}} \\
& =\frac{r}{\left(3-2 r^{2}\right)^{3 / 2}}\left[2 \frac{\varepsilon(r)-\left(1-r^{2}\right) \kappa(r)}{r^{2}}-\frac{\kappa(r)-\varepsilon(r)}{r^{2}}\right] .
\end{aligned}
$$

From (2.2) and Lemma 2.2(1)-(2) that

$\phi^{\prime}(r)>\frac{r}{\left(3-2 r^{2}\right)^{3 / 2}}\left[2 \times \frac{\pi}{4}-2(\kappa(\sqrt{2} / 2)-\varepsilon(\sqrt{2} / 2))\right]>0$

for $r \in(0, \sqrt{2} / 2)$.

Therefore, part (3) follows form (2.1) and (2.3).

For part (4), Let $\varphi(r)=\sqrt{3-2 r^{2}}\left[\varepsilon(r)-\left(1-r^{2}\right) \kappa(r)\right] / r^{2}$. Then simple computations lead to

$\varphi\left(\mathrm{O}^{+}\right)=\frac{\sqrt{3} \pi}{4}, \varphi\left(\frac{\sqrt{2}}{2}^{-}\right)=\sqrt{2}\left[2 \varepsilon\left(\frac{\sqrt{2}}{2}\right)-\kappa\left(\frac{\sqrt{2}}{2}\right)\right]$,

Differentiating $\varphi(r)$ gives

$$
\varphi^{\prime}(r)=\frac{-6 \varepsilon(r)+2 r^{2} \varepsilon(r)+6 \kappa(r)-5 r^{2} \kappa(r)}{r^{3} \sqrt{3-2 r^{2}}},
$$

Let

$\varphi_{1}(r)=-6 \varepsilon(r)+2 r^{2} \varepsilon(r)+6 \kappa(r)-5 r^{2} \kappa(r)$,

$\varphi_{1}\left(0^{+}\right)=0$,

$\varphi_{1}^{\prime}(r)=\frac{7 r^{3}}{1-r^{2}}\left[\frac{\varepsilon(r)-\left(1-r^{2}\right) \kappa(r)}{r^{2}}-\frac{6}{7} \varepsilon(r)\right]$.

From (2.8) and Lemma 2.2(1) together with the monotonicity $\varepsilon(r)$ on $(0,1)$ we get

$\varphi_{1}^{\prime}(r)<\frac{r^{3}}{1-r^{2}}[8 \varepsilon(\sqrt{2} / 2)-7 \kappa(\sqrt{2} / 2)]<0$

for $r \in(0, \sqrt{2} / 2)$.

Therefore, part (4) follows form (2.4)-(2.7) and (2.9).

Lemma 2.3. The function

$\gamma(r)=\frac{\left(2 r^{2}-1\right) \varepsilon(r)+\left(1-r^{2}\right) \kappa(r)}{r^{2} \sqrt{1-r^{2}}}$

is strictly increasing from $(0, \sqrt{2} / 2)$ onto $(3 \pi / 4, \sqrt{2} \kappa(\sqrt{2} / 2))$. 
Proof. Simple computations lead to

$$
\gamma\left(0^{+}\right)=\frac{3 \pi}{4}, \gamma\left(\frac{\sqrt{2}}{2}^{-}\right)=\sqrt{2} \kappa\left(\frac{\sqrt{2}}{2}\right)
$$

Differentiating $\gamma(r)$ gives

$$
\gamma^{\prime}(r)=\frac{\left(2-r^{2}\right) \varepsilon(r)-2\left(1-r^{2}\right) \kappa(r)}{r^{3}\left(1-r^{2}\right)^{3 / 2}} .
$$

Let

$\gamma_{1}(r)=\left(2-r^{2}\right) \varepsilon(r)-2\left(1-r^{2}\right) \kappa(r)$,

Then we get

$\gamma_{1}\left(0^{+}\right)=0$

$\gamma_{1}^{\prime}(r) / 3 r^{3}=\frac{\kappa(r)-\varepsilon(r)}{r^{2}}$.

It from (2.14) and Lemmas 2.2(2) that

$\gamma_{1}^{\prime}(r)>0$

for all $r \in(0, \sqrt{2} / 2)$.

Therefore, Lemma 2.3 follows from (2.10)-(2.13) and (2.15).

Lemma 2.4. The function $\lambda(r)=\frac{r^{2} \varepsilon(r)+\left(1-r^{2}\right)[\kappa(r)-\varepsilon(r)]}{r^{2} \sqrt{1-r^{2}}}$ is strictly increasing from $(0, \sqrt{2} / 2)$ onto $(3 \pi / 4, \sqrt{2} \kappa(\sqrt{2} / 2))$.

Proof. Let $\lambda_{1}(r)=r^{2} \varepsilon(r)+\left(1-r^{2}\right)[\kappa(r)-\varepsilon(r)], \quad \lambda_{2}(r)=r^{2} \sqrt{1-r^{2}}$ and $\lambda(r)=\frac{\lambda_{1}(r)}{\lambda_{2}(r)}=\frac{r^{2} \varepsilon(r)+\left(1-r^{2}\right)[\kappa(r)-\varepsilon(r)]}{r^{2} \sqrt{1-r^{2}}}$.

Then simple computations lead to

$$
\begin{aligned}
& \lambda_{1}\left(0^{+}\right)=\lambda_{2}(0)=0, \\
& \frac{\lambda_{1}^{\prime}(r)}{\lambda_{2}^{\prime}(r)}=\frac{3 \sqrt{1-r^{2}}[2 \varepsilon(r)-\kappa(r)]}{2-3 r^{2}}:=3 \mu(r),
\end{aligned}
$$

where

$$
\begin{aligned}
& \mu(r)=\frac{\sqrt{1-r^{2}}[2 \varepsilon(r)-\kappa(r)]}{2-3 r^{2}} . \\
& \mu\left(0^{+}\right)=\frac{\pi}{4}, \\
& \mu^{\prime}(r)=\frac{2[\varepsilon(r)-\kappa(r)]+r^{2}[\varepsilon(r)+\kappa(r)]}{r \sqrt{1-r^{2}}\left(2-3 r^{2}\right)^{2}} \\
& =\frac{2 r}{\sqrt{1-r^{2}}\left(2-3 r^{2}\right)^{2}}\left[\frac{\varepsilon(r)-\kappa(r)}{r^{2}}+\frac{\varepsilon(r)+\kappa(r)}{2}\right] .
\end{aligned}
$$

From (2.21) and Lemmas 2.2(2) that

$$
\mu^{\prime}(r)>0
$$

foll all $r \in(0, \sqrt{2} / 2)$. 
It follows from (2.18)-(2.22) we clearly see that $\lambda_{1}^{\prime}(r) / \lambda_{2}^{\prime}(r)$ is strictly increasing on $(0, \sqrt{2} / 2)$. Note that

$$
\lim _{r \rightarrow 0^{+}} \lambda(r)=\frac{3}{4} \pi, \lim _{r \rightarrow \frac{\sqrt{2}^{-}}{2}} \lambda(r)=\sqrt{2} \kappa(\sqrt{2} / 2) .
$$

Therefore, Lemma 2.4 follows from Lemma 2.1, (2.17), (2.23), and the monotonicity of $\lambda_{1}^{\prime}(r) / \lambda_{2}^{\prime}(r)$.

\section{MAIN RESUltS}

Theorem 3.1 The double inequality

$$
E^{\alpha_{1}}(a, b) A^{1-\alpha_{1}}(a, b)<T[A(a, b), Q(a, b)]<E^{\beta_{1}}(a, b) A^{1-\beta_{1}}(a, b)
$$

holds for all $a, b>0$ with $a \neq b$ if and only if

$\alpha_{1} \leq[2 \log \varepsilon(\sqrt{2} / 2)+3 \log 2-2 \log \pi] /(4 \log 2-2 \log 3)=0.6798 \mathrm{~L}$ and $\beta_{1} \geq 3 / 4$.

Proof. Since $A(a, b), T(a, b)$ and $E(a, b)$ are symmetric and homogenous of degree 1 .

Without loss of generality, we assume that $a>b>0$. Let $r=(a-b) / \sqrt{2\left(a^{2}+b^{2}\right)} \in(0, \sqrt{2} / 2)$. Then follows from (1.1) and (1.3) lead to

$$
\begin{aligned}
& T[A(a, b), Q(a, b)]=\frac{2 A(a, b)}{\pi \sqrt{1-r^{2}}} \varepsilon(r), \\
& E(a, b)=\frac{A(a, b)\left(3-2 r^{2}\right)}{3\left(1-r^{2}\right)} .
\end{aligned}
$$

Then it is follows from (3.1) and (3.2) lead to

$$
\frac{\log T[A(a, b), Q(a, b)]-\log A(a, b)}{\log E(a, b)-\log A(a, b)}=\frac{\log \left[\frac{2}{\pi} \varepsilon(r)\right]-\log \sqrt{1-r^{2}}}{\log \left(3-2 r^{2}\right)-\log \left[3\left(1-r^{2}\right)\right]} .
$$

Let $f_{1}(r)=\log \left[\frac{2}{\pi} \varepsilon(r)\right]-\log \sqrt{1-r^{2}}, f_{2}(r)=\log \left(3-2 r^{2}\right)-\log \left[3\left(1-r^{2}\right)\right]$ and

$$
f(r)=\frac{f_{1}(r)}{f_{2}(r)}=\frac{\log \left[\frac{2}{\pi} \varepsilon(r)\right]-\log \sqrt{1-r^{2}}}{\log \left(3-2 r^{2}\right)-\log \left[3\left(1-r^{2}\right)\right]} .
$$

Then simple computations lead to

$$
\begin{aligned}
& f_{1}\left(\mathrm{O}^{+}\right)=f_{2}\left(\mathrm{O}^{+}\right)=0, \\
& \frac{f_{1}^{\prime}(r)}{f_{2}^{\prime}(r)}=\frac{\left(3-2 r^{2}\right)\left[\varepsilon(r)-\left(1-r^{2}\right) \kappa(r)\right]}{2 r^{2} \varepsilon(r)} \\
& =\frac{1}{2} \times \frac{\sqrt{3-2 r^{2}}\left[\varepsilon(r)-\left(1-r^{2}\right) \kappa(r)\right] / r^{2}}{\varepsilon(r) / \sqrt{3-2 r^{2}}} .
\end{aligned}
$$

From equation (3.6), and Lemma 2.2(3) and (4) we clearly see that $f_{1}^{\prime}(r) / f_{2}^{\prime}(r)$ is strictly decreasing on $(0, \sqrt{2} / 2)$. Note that

$$
\begin{aligned}
& \lim _{r \rightarrow 0^{+}} f(r)=\lim _{r \rightarrow 0^{+}} \frac{f_{1}^{\prime}(r)}{f_{2}^{\prime}(r)}=\frac{3}{4}, \\
& \lim _{r \rightarrow \frac{\sqrt{2}}{2}} f(r)=\frac{2 \log \varepsilon(\sqrt{2} / 2)+3 \log 2-2 \log \pi}{4 \log 2-2 \log 3}=0.6798 \mathrm{~L} .
\end{aligned}
$$


$E[p a+(1-p) b, p b+(1-p) a]=\frac{A(a, b)}{1-r^{2}}\left[\frac{2}{3}\left(2 p^{2}-2 p-1\right) r^{2}+1\right]$

we get

$$
E[p a+(1-p) b, p b+(1-p) a]-T[A(a, b), Q(a, b)]=\frac{A(a, b)}{1-r^{2}} h(r),
$$

where

$$
h(r)=\frac{2}{3}\left(2 p^{2}-2 p-1\right) r^{2}+1-\frac{2}{\pi} \sqrt{1-r^{2}} \varepsilon(r) .
$$

Then simple computations lead to

$h\left(0^{+}\right)=0$,

$h\left(\frac{\sqrt{2}^{-}}{2}\right)=\frac{2}{3}\left(p^{2}-p+1\right)-\frac{\sqrt{2}}{\pi} \varepsilon\left(\frac{\sqrt{2}}{2}\right)$,

Let

$h_{1}(r)=h^{\prime}(r) /(2 r)$.

Then (3.19) and Lemma 2.4 lead

$$
\begin{aligned}
& h_{1}(r)=\frac{1}{\pi} \frac{r^{2} \varepsilon(r)+\left(1-r^{2}\right)[\kappa(r)-\varepsilon(r)]}{r^{2} \sqrt{1-r^{2}}}+\frac{2}{3}\left(2 p^{2}-2 p-1\right), \\
& h_{1}\left(0^{+}\right)=\frac{1}{12}\left(16 p^{2}-16 p+1\right), \\
& h_{1}\left(\frac{\sqrt{2}^{-}}{2}\right)=\frac{\sqrt{2}}{\pi} \kappa\left(\frac{\sqrt{2}}{2}\right)+\frac{2}{3}\left(2 p^{2}-2 p-1\right) .
\end{aligned}
$$

We divide the proof into four cases.

Case 1. $p=1 / 2+\sqrt{3\left[2 \sqrt{2} \pi \varepsilon(\sqrt{2} / 2)-\pi^{2}\right]} /(2 \pi)$. Then (3.18), (3.21) and (3.22) lead to

$$
\begin{aligned}
& h\left(\frac{\sqrt{2}^{-}}{2}\right)=0, \\
& h_{1}\left(\mathrm{O}^{+}\right)=\frac{2 \sqrt{2} \varepsilon(\sqrt{2} / 2)}{\pi}-\frac{5}{4}=-0.03399 \mathrm{~L}<\mathrm{O}, \\
& h_{1}\left(\frac{\sqrt{2}}{2}^{-}\right)=\frac{\sqrt{2}[2 \varepsilon(\sqrt{2} / 2)+\kappa(\sqrt{2} / 2)]}{\pi}-2=0.0506 \mathrm{~L}>0
\end{aligned}
$$

It follows from (3.19), (3.24), (3.25) and Lemma 2.4 that there exists $r^{*} \in(0, \sqrt{2} / 2)$ such that $h(x)$ is strictly decreasing on $\left(0, r^{*}\right]$ and strictly increasing on $\left[r^{*}, \sqrt{2} / 2\right)$.

Therefore,

$T[A(a, b), Q(a, b)]>E[p a+(1-p) b, p b+(1-p) a]$

for all $a, b>0$ with $a \neq b$ follows from (3.15), (3.17) and (3.23) together with the piecewise monotonicity of $h(x)$. 
Case 2. $p=1 / 2+\sqrt{3} / 4$. Then (3.21) becomes

$h_{1}\left(0^{+}\right)=0$,

From Lemma 2.4 and (3.20) we know that $h_{1}(x)$ is strictly increasing on $(0, \sqrt{2} / 2)$ and

$h_{1}(r)>h_{1}\left(0^{+}\right)=0$,

for all $(0, \sqrt{2} / 2)$. Therefore,

$T[A(a, b), Q(a, b)]<E\left[\left(\frac{1}{2}+\frac{\sqrt{3}}{4}\right) a+\left(\frac{1}{2}-\frac{\sqrt{3}}{4}\right) b,\left(\frac{1}{2}+\frac{\sqrt{3}}{4}\right) b+\left(\frac{1}{2}-\frac{\sqrt{3}}{4}\right) a\right]$

for all $a, b>0$ with $a \neq b$ follows from (3.15), (3.17), (3.19) and (3.27).

Case 3. $1 / 2+\sqrt{3\left[2 \sqrt{2} \pi \varepsilon(\sqrt{2} / 2)-\pi^{2}\right]} /(2 \pi)<p<1$. Then (3.18) leads to

$h\left(\frac{\sqrt{2}^{-}}{2}\right)>0$.

Equations (3.15) and (3.28) imply that there exists $0<\delta_{1}<\sqrt{2} / 2$ such that

$T[A(a, b), Q(a, b)]<E[p a+(1-p) b, p b+(1-p) a]$

for all $a, b>0$ with $|a-b| / \sqrt{2\left(a^{2}+b^{2}\right)} \in\left(\sqrt{2} / 2-\delta_{1}, \sqrt{2} / 2\right)$.

Case 4. $1 / 2<p<1 / 2+\sqrt{3} / 4$. Then equation (3.21) leads to

$h_{1}\left(0^{+}\right)<0$.

Equations (3.15), (3.17), and (3.19) and inequality (3.29) imply that there exists

$0<\delta_{2}<\sqrt{2} / 2$ such that

$T[A(a, b), Q(a, b)]>E[p a+(1-p) b, p b+(1-p) a]$

for all $a, b>0$ with $|a-b| / \sqrt{2\left(a^{2}+b^{2}\right)} \in\left(0, \delta_{2}\right)$.

Therefore, Theorem 3.3 follows from Case 1 to 4 .

As an application, Corollary 3.4 follows immediately from Theorems 3.1-3.3. We establish three new inequalities for the complete elliptic integral of second kind.

Corollary3.4. Let $\quad \alpha_{1}=[2 \log \varepsilon(\sqrt{2} / 2)+3 \log 2-2 \log \pi] /(4 \log 2-2 \log 3)=0.6798 \mathrm{~L}$ ， $\beta_{1}=3 / 4, \alpha_{2}=[6 \sqrt{2} \varepsilon(\sqrt{2} / 2)] / \pi-3=0.6480 \mathrm{~L}, \beta_{2}=3 / 4$,

$\alpha_{3}=1 / 2+\sqrt{3\left[2 \sqrt{2} \pi \varepsilon(\sqrt{2} / 2)-\pi^{2}\right]} /(2 \pi)=0.9024 \mathrm{~L}$ and $\beta_{3}=1 / 2+\sqrt{3} / 4$. Then the double inequalities

$\frac{\pi}{2}\left(1-2 r^{2} / 3\right)^{\alpha_{1}}\left(\sqrt{1-r^{2}}\right)^{1-2 \alpha_{1}}<\varepsilon(r)<\frac{\pi}{2}\left(1-2 r^{2} / 3\right)^{\beta_{1}}\left(\sqrt{1-r^{2}}\right)^{1-2 \beta_{1}}$,

$\frac{\pi}{2}\left[\alpha_{2} \frac{\left(3-2 r^{2}\right)}{3 \sqrt{1-r^{2}}}+\left(1-\alpha_{2}\right) \sqrt{1-r^{2}}\right]<\varepsilon(r)<\frac{\pi}{2}\left[\beta_{2} \frac{\left(3-2 r^{2}\right)}{3 \sqrt{1-r^{2}}}+\left(1-\beta_{2}\right) \sqrt{1-r^{2}}\right]$, 


$$
\frac{\pi\left[2\left(2 \alpha_{3}^{2}-2 \alpha_{3}-1\right) r^{2}+3\right]}{6 \sqrt{1-r^{2}}}<\varepsilon(r)<\frac{\pi\left[2\left(2 \beta_{3}^{2}-2 \beta_{3}-1\right) r^{2}+3\right]}{6 \sqrt{1-r^{2}}} .
$$

for all $r \in(0, \sqrt{2} / 2)$.

\section{ACKNOWLEDGMENTS}

This research was supported by the Natural Science Foundation of China under Grants 11371125, 11401191, and 61374086, the Natural Science Foundation of Zhejiang Province under Grant LY13A010004, the Natural Science Foundation of HuZhou City under Grant 2018YZ07, the Natural Science Foundation of Zhejiang Broadcast and TV University under Grant XKT-17Z04 and 17G26.

\section{REFERENCES}

[1] S. H. Wu, A new sharpened and generalized version of Hölder's inequality and its applications, Appl. Math. Comput., 2008, 197, 708-714.

[2] S. H. Wu, L. Debnath, Inequalities for differences of power means in two variables, Anal. Math., 2011, 37, 151-159.

[3] G. Toader, Some mean values related to the arithmetic-geometric mean, J. Math. Anal.Appl., 1998, 218(2), 358-368.

[4] F. Bowmn, Introduction to elliptic functions with applictions, Dover Publictions, New York, 1961.

[5] P. F. Byrd and M. D. Friedmn, Handbook of elliptic integrals for engoneers and scientists, Springer-Verlag, New York, 1971.

[6] G. D. Anderson, M. K. Vamanamurthy, and M. K. Vuorinen, Conformal Invariants, Inequalities, and Quasiconformal Maps, John Wiley \& Sons, New York, 1997.

[7] J. M. Borwein, P. B. Borwein, Pi and the AGM, John Wiley\& Sons, New York, 1987.

[8] E. Neuman, Bounds for symmetric elliptic integrals, J. Approx. Theory, 2003, 122(2), 249-259.

[9] H. Kazi and E. Neuman, Inequalities and bounds for elliptic integrals, J. Approx. Theory,2007, 146(2), 212-226.

[10] H. Kazi and E. Neuman, Inequalities and bounds for elliptic integrals II, In: Special Functions and Orthogonal Polynomials, 127-138, Contemp. Math. 471, Amer. Math. Soc., Providence, 2008, 127-138.

[11] Y. -M. Chu, M. -K. Wang and S.-L. Qiu, Optimal combinations bounds of root-square and arithmetic means for Toader mean, Proc Indian Acad. Sci. Math.Sci., 2012. 122(1): 41-51.

[12] Y. -M. Chu and M. -K. Wang, Optimal Lehmer mean bounds for the Toader mean, Results Math., 2012, 61(3-4): 223-229.

[13] Y. -M. Chu, M. -K. Wang and X.-Y. Mo, Sharp bounds for Toader mean in terms of contrahrmonic mean with applications, J. Math. Inequalities. 2013.7(2): 161 -166.

[14] J.-F. Li, W.-M. Qian and Y. -M. Chu, Sharp bounds for Toader mean in terms of arithmetic, quadratic, and Neuman means, J. Inequal. Appl., 2015, 2015: 277.

[15] Y. -Q. Song, W. -D. Jiang, Y. -M. Chu and D. -D. Yan, Optimal bounds for Toader mean in terms of arithmetic and contrahrmonic means, J. Math. Inequalities. 2013.7(4): 751 -777.

[16] G. D. Anderson, S.-L. Qiu, M. K. Vamanamurthy, and M. Vuorinen, Generalized elliptic integrals and modular equations, Pacific J. Math., 2000, 192(1), 1-37.

[17] M. Vuorinen, Hypergeometric functions in geometric function theory, In: Special functions and differential equations (Madras, 1977), 119-126, Allied Publ., New Delhi, 1998.

[18] S.-L. Qiu and J.-M. Shen, On two problems concerning means, J. Hangzhou Inst. Electron. Eng., 1997, 17(3), 1-7 (in Chinese).

[19] R. W. Barnard, K. Pearce and K. C. Richards, An inequality invoving the generalized hypergeometric function and the arc length of an ellipse, SIAM J. Math. Anal., 2000, 31(3),693-699. 
[20] Alzer H and Qiu S-L, Monotonicity theorems and inequalities for the complete elliptic integrals, J. Comput. Appl. Math. 172(2) (2004) 289-312.

[21] Y. Hua and F. Qi, The best bounds for Toader mean in terms of the centroidal and arithmetic means, Filomat, 2014, 28(4): 775-780.

[22] Y. Hua and F. Qi, A double inequality for bounding Toader mean by the centroidal mean, Proc. Indian Acad. Sci. Math. Sci., 2014, 124(4), 527-531.

[23] H.-Z. Xu, W.-M. Qian, Sharp bounds for Toader-type mean by other classic bivariate means (Chinese), Mathematics in Practice and Theory, 2017, 47(3), 288-296.

[24] T.-H. Zhao, Y.-M. Chu and W. Zhang, Optimal inequalities for bounding Toader mean by arithmetic and quadratic means, J. Inequal. Appl., 2017, 2017: 26.

[25] H.-H. Chu, W.-M. Qian, Y.-M. Chu, Y.-Q. Song, Optimal bounds for a Toader-type mean in terms of one-parameter quadratic and contraharmonic means, J. Nonlinear Sci. Appl. 9 (2016), 3424-3432.

Citation: Yue-Ying Yang et.al., (2019). Optimal Bounds for Toader-Type Mean in Terms of Arithmetic and Centroidal Means. International Journal of Scientific and Innovative Mathematical Research (IJSIMR), 7(2), pp. 35-45. http://dx.doi.org/10.20431/2347-3142.0702005

Copyright:@ 2019Authors, This is an open-access article distributed under the terms of the Creative Commons Attribution License, which permits unrestricted use, distribution, and reproduction in any medium, provided the original author and source are credited. 